\title{
Oral steroids enhance epithelial repair in nasal polyposis via upregulation of the AP-1 gene network
}

\author{
C W Li, ${ }^{1}$ W Cheung, ${ }^{2}$ Z B Lin, ${ }^{3}$ T Y Li, ${ }^{3}$ J T Lim, ${ }^{4}$ D Y Wang'
}

\begin{abstract}
- Additional methods information is published online only at http://thorax.bmj.com/ content/vol64/issue4

${ }^{1}$ Department of Otolaryngology, Yong Loo Lin School of

Medicine, National University of Singapore, Singapore;

${ }^{2}$ Department of Pediatrics,

University of California San

Diego, La Jolla, California;

${ }^{3}$ Department of

Otorhinolaryngology, The First

Affiliated Hospital, Sun Yet-Sen

University, Guangzhou, China;

${ }^{4}$ Department of Pathology, Yong

Loo Lin School of Medicine,

National University of Singapore,

Singapore
\end{abstract}

Correspondence to:

Dr D Y Wang, Department of

Otolaryngology, Yong Loo Lin

School of Medicine, National

University of Singapore, 10

Lower Kent Ridge Road,

Singapore 119260; entwdy@

nus.edu.sg

Received 14 August 2008

Accepted 16 December 2008

Published Online First

20 January 2009

\begin{abstract}
Background: Chronic mucosal inflammation, epithelial damage and aberrant tissue remodelling are common features in nasal polyposis (NP). A study was undertaken to characterise the gene expression profile in NP tissues and to explore the molecular mechanisms underlying the ameliorative effects of glucocorticosteroids (GCs) in NP.

Methods: Two sets of NP biopsies (before and after GC treatment) were taken from 10 patients with untreated (GC-naïve) bilateral NP. Biopsy specimens of inferior turbinate from 6 patients who underwent surgery for nasal septal deviation served as nasal mucosal controls. DNA microarrays containing 38500 genes were used to characterise the global gene expression profile. Functional network analysis was applied to identify the key molecular pathways and genes in response to GC treatment (GCtreated). Selected genes were retested by quantitative RT-PCR and immunohistochemistry in the same polyps and control samples.
\end{abstract}

Results: 64 genes were differentially expressed in GCtreated vs GC-naive NP tissues. The highest scoring network was assembled around activation protein 1 (AP1), a heterodimer of c-Fos and c-Jun oncoprotein, and five AP-1-related genes (COX-2, IL-6, AREG, HBEGF and EGR1) with tissue repair function. Quantitative PCR confirmed that AP-1 and its related genes were markedly repressed in GC-naive polyps and were upregulated after GC treatment. Immunohistochemical staining indicated that epithelial restitution in GC-treated polyps was associated with increased expression of c-Jun protein.

Conclusions: Oral steroids promote epithelial repair in NP via upregulation of the AP-1 (especially c-Jun) network and its related genes.

Nasal polyposis (NP), a common chronic inflammatory disorder of the upper airways, is often associated with asthma and other respiratory diseases. ${ }^{1}$ The infiltration of various inflammatory cells, epithelial damage and expression of the Th2 cytokines are similar to the pathological profile of asthma. From this perspective, NP could therefore be regarded as a paradigm of chronic airway inflammation. ${ }^{2}$

The important features of NP are mucosal inflammation, epithelial damage followed by aberrant tissue repair and structural changes (remodelling). ${ }^{3}$ Inflammation in NP is characterised by the increased expression of multiple inflammatory genes regulated by proinflammatory transcription factors such as nuclear factor (NF)- $\mathrm{KB}$ and activator protein-1 (AP-1), which binds to and activates coactivator molecules that acetylate core histones and switch on gene transcription. Enhanced immune and inflammatory responses in NP are associated with tissue repair and remodelling in the target tissue and are related to disease severity Recruited inflammatory cells and activated epithelial cells in NP are the major source of cytokines sustaining the inflammatory reaction and activation, proliferation of fibroblasts and myofibroblast differentiation.

Glucocorticosteroids (GCs) are the most effective anti-inflammatory therapy for NP. ${ }^{1}$ The ability of GCs to elicit an anti-inflammatory effect is mediated by suppressing the multiple inflammatory genes that are activated in NP, mainly by reversing histone acetylation of activated inflammatory genes through binding of glucocorticoid receptors to coactivators and recruitment of histone deacetylase 2 to the activated transcription complex. ${ }^{4}$ An inflammatory insult could lead to normal reconstruction or pathological remodelling. An important aspect of GCs is to promote tissue repair and remodelling in NP and asthma through transforming growth factor (TGF)- $\beta$ and epidermal growth factor/epidermal growth factor receptor (EGF/EGFR) signalling pathways. ${ }^{5}$ Previous studies have shown that transcription factors, cytokines and growth factors also influence the wound healing process in airway tissues. ${ }^{7-9}$

Airway epithelial repair is a well coordinated process. Epithelial remodelling in NP is a complex process which includes migration, proliferation and differentiation of epithelial cells, involving groups of interacting molecules such as transcription factors, cytokines and growth factors in overlapping signalling networks. In this study we sought to characterise the genome-wide transcriptional profile within NP tissues. We also sought to explore the genome-wide interaction network and to identify significant functional modules perturbed in response to GC therapy by comparing dysregulation of functional modules in NP before and after oral GCs. Understanding the mechanism by which GCs restore the normal phenotypic appearance within the nasal cavity may yield clues to the pathobiology of NP and enable the development of novel treatments.

\section{METHODS}

\section{Nasal tissues}

Ten patients with NP with refractory nasal obstruction underwent functional endoscopic sinus surgery. Two sets of polyp biopsies were taken from the same patient (before (GC-naïve) and after (GC-treated) initiating treatment with oral prednisone $(10 \mathrm{mg}$ three times per day for 3-5 days). None of the patients had an upper respiratory infection nor had they taken any form of GCs or antibiotics for more than 3 months before the study. None of the subjects had a 
Table 1 Clinical characteristics of patients undergoing polypectomy surgery for nasal polyposis (NP) or inferior turbinectomy (IT) for septal deviation (SD)

\begin{tabular}{|c|c|c|c|c|c|c|c|c|}
\hline \multirow[b]{2}{*}{ Code } & \multirow{2}{*}{$\begin{array}{l}\text { Age } \\
\text { (years) }\end{array}$} & \multirow[b]{2}{*}{ Sex } & \multirow[b]{2}{*}{ Diagnosis } & \multirow[b]{2}{*}{ Atopy } & \multicolumn{2}{|l|}{ c-Jun staining* } & \multicolumn{2}{|l|}{ Epithelial damage $\uparrow$} \\
\hline & & & & & Before treatment & After treatment & Before treatment & After treatment \\
\hline \multicolumn{9}{|c|}{ Nasal polyposis } \\
\hline NP3 & 18 & M & NP & + & 9 & 9 & 0 & 0 \\
\hline NP4 & 55 & $\mathrm{~F}$ & NP & + & 2 & 9 & 2 & 0 \\
\hline NP5 & 32 & $M$ & NP & + & 6 & 9 & 2 & 0 \\
\hline NP7 & 34 & $\mathrm{M}$ & NP & + & 9 & 9 & 1 & 0 \\
\hline NP8 & 55 & $\mathrm{M}$ & NP & + & 9 & 9 & 0 & 0 \\
\hline NP9 & 30 & M & NP & - & 4 & 9 & 1 & 0 \\
\hline NP10 & 24 & $\mathrm{M}$ & NP & + & 4 & 9 & 1 & 1 \\
\hline \multicolumn{9}{|c|}{ Nasal mucosal controls } \\
\hline IT1 & 26 & M & SD & - & NA & NA & NA & NA \\
\hline IT6 & 27 & M & SD & + & 9 & NA & 0 & NA \\
\hline
\end{tabular}

NA, not applicable.

${ }^{*} \mathrm{C}$-Jun staining is described in the online supplement; overall score obtained by multiplying the extent score $(0-3)$ by the intensity score $(0-3)$.

$\dagger$ Epithelium evaluation as described in the online supplement: 0 , intact epithelium; 1, moderately damaged epithelium; 2, severely damaged epithelium.

history of aspirin exposure or asthma. Cases were coded to provide confidentiality. The clinical characteristics, diagnosis and atopic status (determined by serum specific IgE levels) of the subjects were noted (table 1). Biopsy tissue from the inferior turbinate was taken from six control subjects who underwent surgery for nasal septal deviation. This tissue served as a nasal mucosal control. Fresh specimens were divided into two sections; one section was preserved with RNAlater (Ambion, Austin, TX, USA) for gene expression profiling and the other section was fixed in formalin for histological evaluation.

\section{Microarray study and ingenuity pathways analysis (IPA)}

Total cellular RNA was isolated from nasal tissues with RiboPure Kit (Ambion). The concentration and integrity of the purified RNA was determined. cRNA was reversely transcribed using $1 \mu \mathrm{g}$ total RNA. Synthesised cRNA was hybridised onto Human Genome U133 Plus 2.0 arrays (Affymetrix, Santa Clara, CA, USA) and processed accordingly (http://www.affymetrix.com/Auth/support/downloads/manuals/expression_ever_manual.zip). The quality of the microarray data was assessed (https://support/downloads/manuals/ data_analysis_fundamentals_manual.pdf). This led to the exclusion of two NP samples and one control sample for microarray study. A total of 54000 probe sets, representing approximately 38500 genes, was analysed. Details of the analysis are shown in the online supplement. Functional and network analysis were performed using ingenuity pathways analysis (IPA) tools (Ingenuity Systems, www.ingenuity.com). Complete information is available in the online supplement.

\section{Quantitative PCR}

Selective genes were validated with quantitative PCR by using ABI Prism 7000 Sequence Detection System (ABI Applied Biosystems, Foster City, CA, USA). Relative gene expression was calculated using the comparative $2^{-\Delta \Delta C t}$ method $^{10}$ with glyceraldehyde 3-phosphate dehydrogenase (GAPDH) as a reference.

\section{Histological evaluation}

Paraffin sections of nasal biopsies were stained with haematoxylin and eosin and eosinophil infiltration and the epithelial integrity of the biopsies were evaluated. ${ }^{11}$ c-Jun and c-Fos proteins were stained with monoclonal antibodies (Dako A/S, Glostrup, Denmark) and evaluated using a semiquantitative scoring system. ${ }^{12}$ Detailed information is provided in the online supplement.

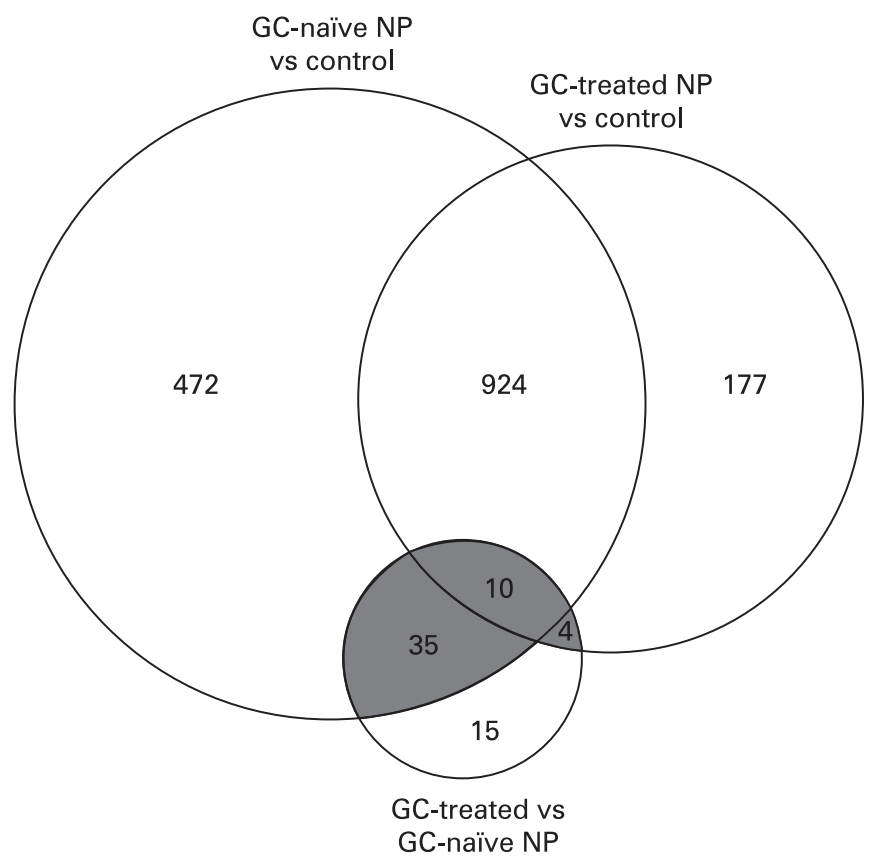

Figure 1 Venn diagram showing overlapping of differentially expressed genes in glucorticosteroid (GC)-naïve nasal polyposis (NP) tissue vs nasal mucosal control, GC-treated NP tissue vs nasal mucosal control and GC-treated vs GC-naïve NP tissue. 


\section{Statistical analysis}

Power sample estimation suggested that a minimum of four biopsies in each study group were required to detect a twofold difference in gene expression with a $1 \%$ significance level and $90 \%$ power (data not published). We would therefore expect to detect a twofold difference in gene expression with $p<0.01$ and with a $90 \%$ chance of avoiding a type II error. Median values of fold change of gene expression were analysed using SPSS Version 14 (SPSS, Chicago, Illinois, USA). The Wilcoxon matched pairs sign rank test was used to compare gene expression levels between GC-treated and GC-naïve NP tissue. The Mann-Whiney two-tailed test was performed to compare gene expression levels between NP tissues (either GC-treated or GC-naïve) and nasal mucosal controls. Spearman rank analysis was used to analyse the correlation between the gene expression levels assessed by microarray and those measured by quantitative PCR, and between fold change of c-Jun and other AP-1 related genes. The Fisher exact test was used to assess the protein expression of c-Jun and the epithelial integrity in NP tissues. p Values $<0.05$ were considered significant.

\section{RESULTS}

\section{Genome-wide transcriptional alterations}

Genome-wide transcriptional alterations were measured and analysed. Significant changes were detected in 2482 transcripts that were differentially expressed in GC-naïve NP tissues compared with nasal mucosal controls, while 1908 transcripts differed in relative expression in GC-treated NP tissues compared with nasal mucosal controls (data not shown). The difference in the relative intensities of all these transcripts was at least twofold. For GC-treated versus GC-naïve NP tissues, 103 differentially regulated transcripts were detected. After filtering the unmapped genes, genes with unknown gene ontology and genes with redundant probe identities, 1441 and 1115 genes were found to be differentially expressed in GCnaïve and GC-treated tissues, respectively, compared with nasal mucosal controls (fig 1). The expression of 64 genes was

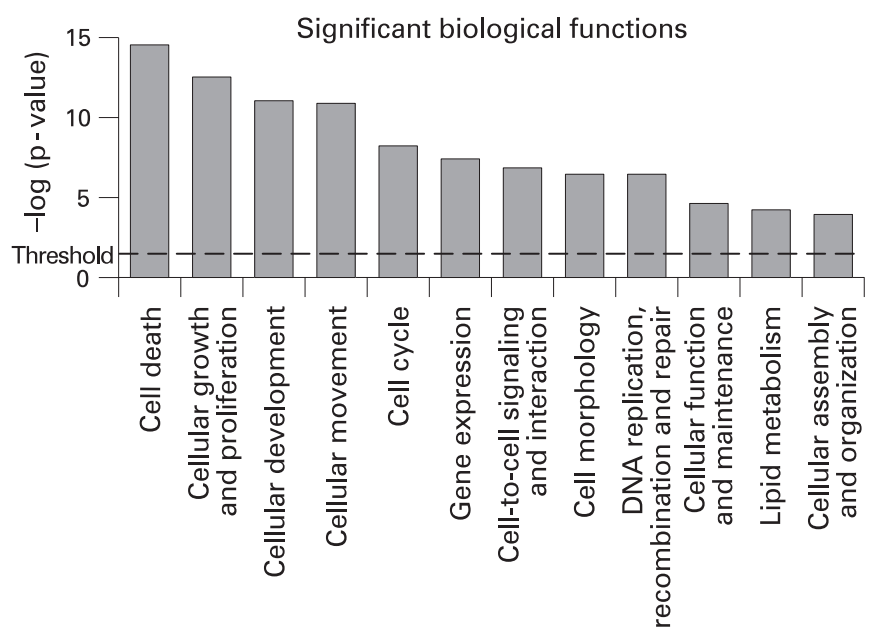

Figure 2 Twelve significant functional annotations for gene sets differentially expressed in glucocorticosteroid (GC)-treated versus GCnaïve nasal polyposis (NP) tissues; $p$ values for a given function were calculated by considering the number of functional analysis genes participating in that function and the total number of genes known to be associated with that function in the ingenuity pathway knowledge base (IPKB). On the y axis, the significance is expressed as the exponent of the $p$ value calculated for each function. The horizontal line represents the threshold $(p=0.05)$ of the significant level of the functions. different in GC-treated NP tissue compared with GC-naïve NP tissue (fig 1 and table E1 in online supplement). Interestingly, among those 64 GC-responsive genes, most of them ( $n=45$, $70.3 \%$ ) were also differentially expressed in GC-naïve NP tissue compared with nasal mucosal controls.

\section{Integrated analysis of molecular pathways}

Sixty-four differentially expressed GC-responsive genes were further analysed using IPA into functional categories and pathways. Our results indicated that differentially expressed molecules regulate critical biological functions such as cell death (43 molecules, $p=3.58 \times 10^{-15}$ ), cellular growth and proliferation (47 molecules, $p=4.45 \times 10^{-13}$ ), cellular development (36 molecules, $p=1.51 \times 10^{-11}$ ) and cellular movement (34 molecules, $p=1.94 \times 10^{-11}$; fig 2 ).

To further elucidate the global changes in NP tissue and its response to GC treatment, we sought to computationally decipher the principal networks involved. The specificity of the connections for each gene was calculated, as defined by the percentage of its direct connections to other genes showing significant transcriptional changes. Our global representation revealed concerted dysregulation of functional modules in NP tissue. Among four networks identified by IPA calculation, the AP-1 network had the top score (39). Notably, 18 of the 64 GCresponsive genes that were differentially expressed had at least one gene in the AP-1 pathway, underscoring its pivotal role in its responsiveness to GC therapy (see table E2 in the online supplement). At the level of interconnecting functional modules, two important AP-1 genes (c-Jun and c-Fos) were the prominent interaction partners (see fig 3 and fig E1 in the online supplement). In addition, evaluation of the AP-1 subnetwork regions revealed that a group of AP-1 related genes (COX-2, IL6, AREG, HBEGF and EGR1) were modulated in NP tissue by the administration of GCs (see table E2 in the online supplement).

\section{Quantitative PCR}

To verify the microarray results, the same starting materials were used for quantitative PCR measurements. Appropriate primers for the genes of interest and controls were used (see table E3 in online supplement). The PCR data for AP-1 and its related genes were comparable to the results generated by the microarray experiments (fig 4). The rank order and magnitude of the gene expression profile derived from quantitative PCR were in accordance with the microarray data. Two glucocorticoid receptor (GR) isoforms were also measured by quantitative PCR; GR $\alpha$ mRNA was significantly decreased in NP tissue compared with control tissues and GR $\beta$ mRNA was undetectable in all samples (data not shown).

We investigated the correlation among gene expression in GC-treated NP tissues. c-Jun mRNA was positively correlated with c-Fos and JunB mRNA $(r=0.924, \mathrm{p}<0.001$ and $r=0.827$, $p=0.003$, respectively; fig $5 \mathrm{~A}, \mathrm{~B})$. Similarly, c-Jun gene expression was positively correlated with AP-1-related genes (COX-2, IL-6 and EGR1) in GC-treated NP tissues (COX-2, $r=0.827, \mathrm{p}=0.003 ; \mathrm{IL}-6, \quad r=0.662, \mathrm{p}=0.03$ and EGR1, $r=0.893, \mathrm{p}=0.001$; fig $5 \mathrm{~A}-\mathrm{C})$.

\section{Histological and immunohistochemical studies}

Significant epithelial damage was evident in 8 of 10 GC-naïve NP tissues (table 1). Subtle epithelial damage (ie, squamous metaplasia) was also detected in the other 2 patients (NP3 and NP8 in table 1). The decrease in eosinophil infiltration in 


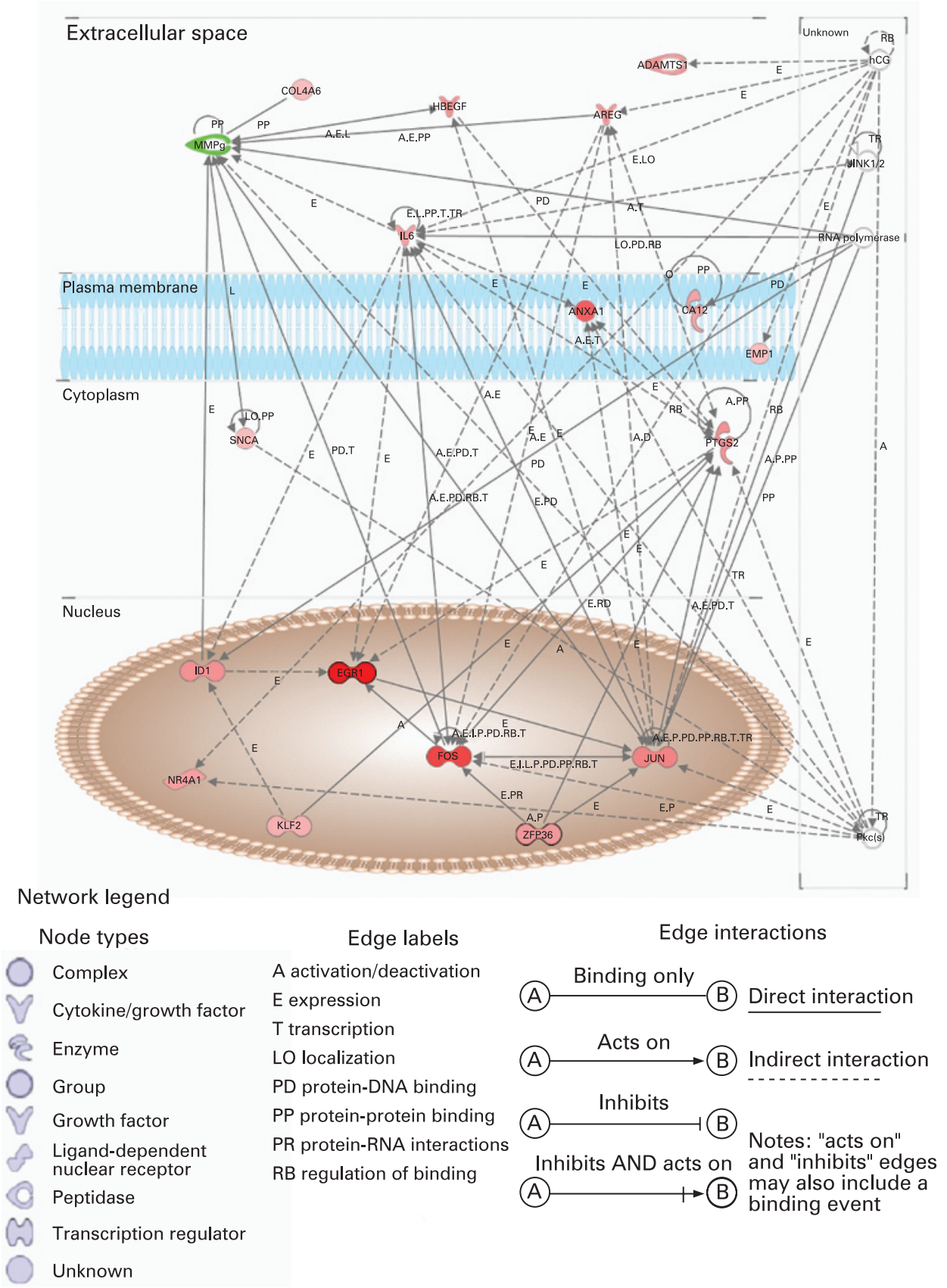

Figure 3 Functional network analysis. The top scoring network composed of glucocorticosteroid (GC)-responsive genes was complemented by an unsupervised relevance network learning algorithm without any prior assumptions and after adjustment. The molecules in this network are arranged into their various subcellular compartments: nucleus, cytoplasm, plasma membrane and extracellular space. An unknown category also exists for molecules for which no subcellular localisation information is currently available. Nodes represent genes, with their shape representing the functional class of the gene product, and edges indicate the biological relationship between the nodes (see network legend). Nodes are colour-coded according to their $d$ score generated by SAM (red, overexpression; green, underexpression) and the colour intensity increases with the magnitude of altered regulation. PTGS2, the official symbol of COX-2.

GC-treated NP tissue was considerable. In 8 of 10 GC-treated NP tissues, significant improvement in epithelium structure was observed (table 2). Improvement in epithelium structure (ie, absence of squamous metaplasia) was also evident in patients NP3 and NP8 after GC treatment. Immunohistochemical staining indicated that c-Jun protein was predominantly expressed in the epithelium. The c-Jun protein level was markedly lower in GC-naïve NP tissues than in GC-treated NP tissues (fig 6, table 2). Equally important, the c-Jun protein level was positively correlated to the epithelial integrity in both
GC-naïve and GC-treated NP tissues (table 2). No difference was observed in the c-Fos protein level in GC-naive NP tissue, GC-treated NP tissue and nasal mucosal controls (data not shown).

\section{DISCUSSION}

Airway epithelium remodelling may represent a repair response to pathological insults such as inflammation, allergens, mechanical damage and environmental pollutants. Oral GCs are currently the most effective anti-inflammatory medications 


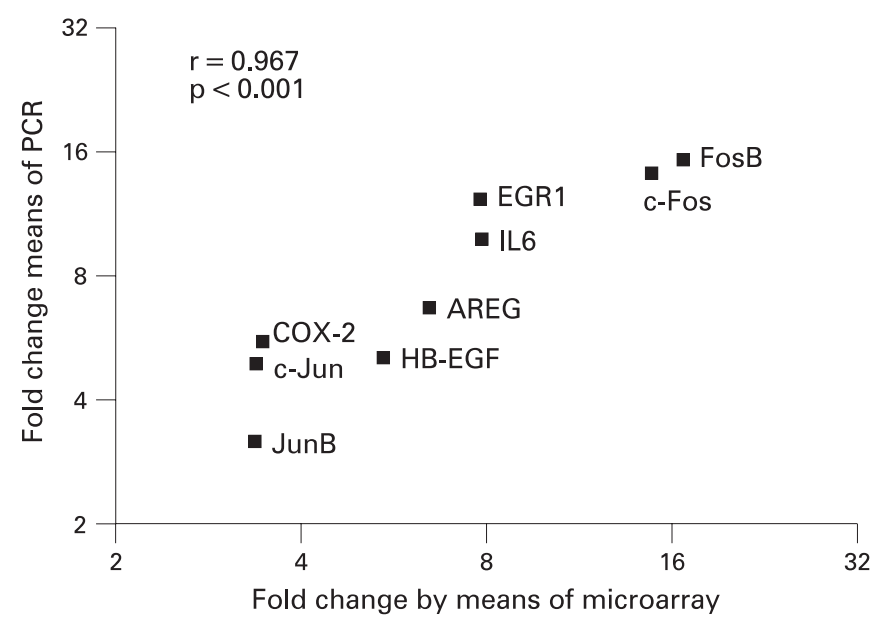

Figure 4 Correlation between quantitative PCR and microarray results. Fold changes were determined by means of PCR and microarray, respectively. Correlations are shown for C-Fos, c-Jun, FosB, JunB, COX2, IL6, HB-EGF, AREG and EGR1. for the treatment of recurrent NP. ${ }^{1}$ This is the first in vivo study to decipher the role of the AP-1 gene pathway and epithelial remodelling in NP. The key findings are: (1) Epithelial damage is an important feature of NP and a remarkable epithelial restitution is observed after GC treatment; (2) AP-1 and its related genes appear to be the key molecular network underlying wound healing in NP; (3) Epithelial c-Jun protein expression is upregulated by oral steroid therapy and is positively correlated with epithelium restitution in NP tissues.

AP-1 is a heterodimer of Fos and Jun oncoproteins. The most abundant AP-1 heterodimer is c-Fos:c-Jun. AP-1 and its related genes participate in the induction of a wide variety of proinflammatory proteins, receptors and mediators. ${ }^{13}$ In this respect, AP-1 induces a variety of growth-related genes (eg, the EGF protein family), ${ }^{14}$ cytokines (eg, IL-6) ${ }^{15}$ and enzymes (eg, PTGS2, known as COX-2). ${ }^{16}$ Recent evidence suggests a causative relationship between AP-1 and epithelial remodelling in airway diseases. ${ }^{14}{ }^{17}$ The epithelial localisation suggests that AP-1 may play an important role in the pathology of NP. We found a significant low level of c-Jun and c-Fos mRNA expression in GC-naïve NP tissues compared with nasal mucosal
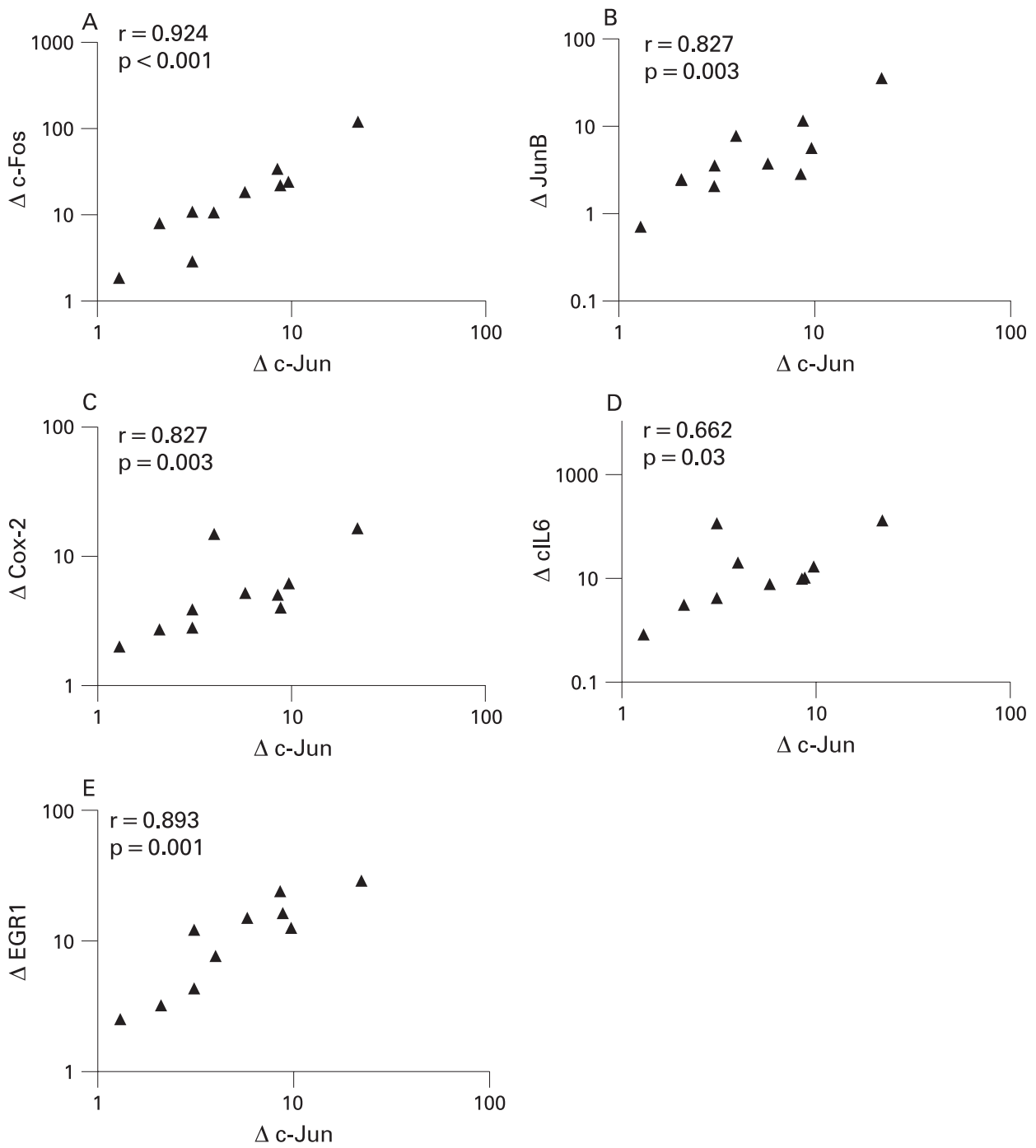

Figure 5 Relationship between mRNA level of c-Jun vs (A) c-Fos, (B) JunB, (C) COX-2, (D) IL6 and (E) EGR1. $\Delta$, fold change in the indicated gene in an individual patient before vs after glucocorticosteroid treatment. Fold changes were determined by PCR. 

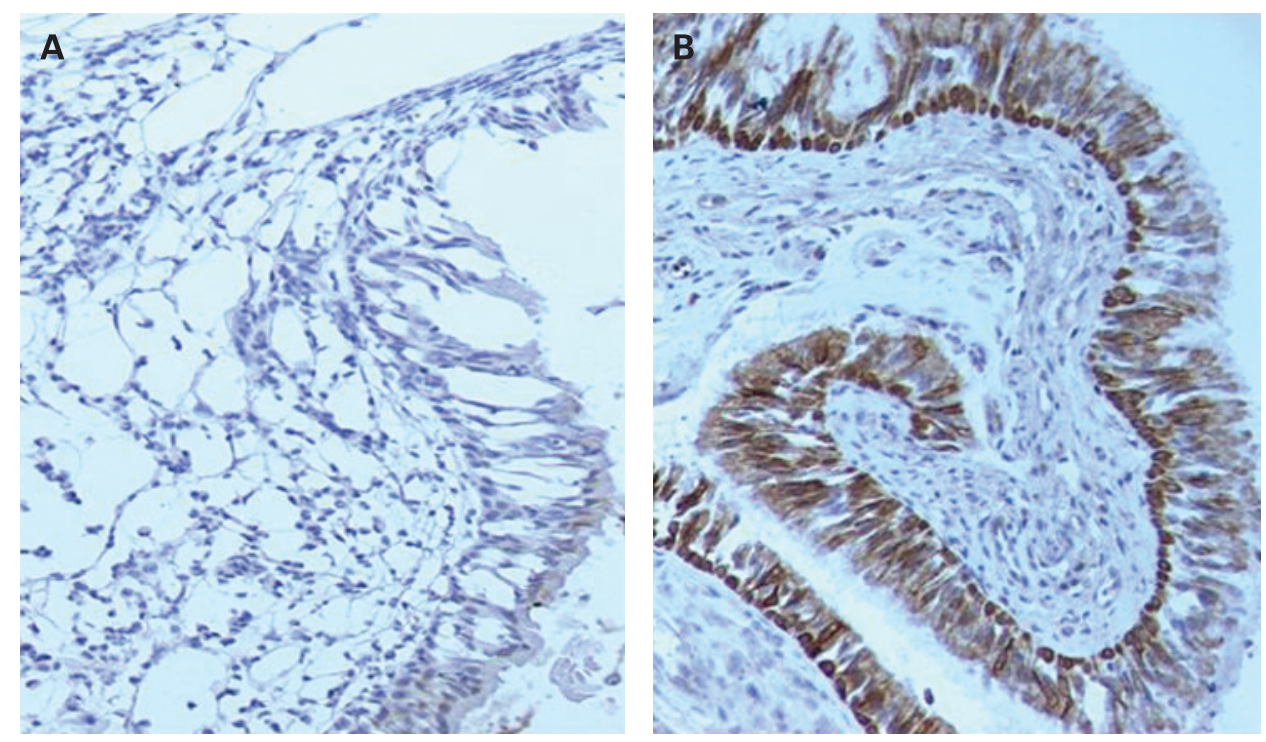

Figure 6 Expression of c-Jun protein determined by immunohistochemistry in a representative patient with nasal polyposis (A) before and (B) after treatment with glucocorticosteroid. Original magnification $\times 200$.

controls. c-Jun mRNA and protein levels were increased after GC therapy in NP tissue. In this study, c-Fos mRNA was upregulated in GC-treated NP. Immunohistochemical staining suggests that GC treatment did not improve the c-fos protein level in extracted NP tissues. Our results are in disagreement with a previous report in which raised c-Fos protein levels were detected in NP tissue and steroid treatment decreased the c-Fos protein level but did not affect the c-Fos mRNA level in NP tissues. ${ }^{18}$ Induction of AP-1 in response to GC treatment is highly complex and may be cell type-specific. ${ }^{19} 20$ c-Fos protein was expressed in endothelial cells, lymphocytes, granulocytes and monocytes. Baraniuk et al have studied the c-Fos mRNA level using a qualitative rather than quantitative measurement. ${ }^{18}$ The c-Fos mRNA level was normalised by $\beta$-actin intensity band, which is not a reliable internal control for GCtreated nasal samples. ${ }^{21}$ c-Fos mRNA and protein expression may also be affected by broad alterations in post-transcriptional processes such as increased turnover of c-Fos protein, negative autoregulation of c-Fos expression and mutual degradation between activated GR and c-Fos. ${ }^{22}{ }^{23}$ Other plausible explanations for the conflicting result include variable factors such as choice of different subjects, dosage and duration of GC treatment, different experimental protocols and tissue types used in the experiments.

The integrated analysis of multidimensional transcriptional data has proved informative. Our network analyses have identified a number of AP-1-related genes (COX-2, IL6, AREG, HBEGF and EGR1) which appeared to modulate airway epithelial remodelling. AP-1 induces transcription of COX-2 which is the key enzyme required for the conversion of arachidonic acid into prostaglandins (PGs). COX-2/PGE signalling regulates the epithelial remodelling process. ${ }^{24}$ Decreased COX-2/PGE 2 has been associated with aspirin intolerance in patients with NP. ${ }^{25}$ We found an upregulation of COX-2 mRNA in NP tissues after oral GC treatment. Dworski et al have shown that treatment with prednisone upregulates COX-2 mRNA and protein levels in atopic subjects. ${ }^{26}$ IL-6 is an important AP-1related gene. AP-1 induces IL- 6 expression through the CRE binding region, ${ }^{27}$ while the IL-6-mediated signal pathway enhances c-Jun expression. ${ }^{28}$ Inhaled corticosteroid promotes IL6 production and favours respiratory epithelial cell remodelling in airway diseases. ${ }^{1529}$ EGFR and its ligands (AREG and HBEGF) are direct AP-1 target genes..$^{30}$ Activation of EGF/EGFR signalling leads to the induction of AP-1. ${ }^{14}{ }^{17}$ The EGF/EGFR signalling pathway influences epithelial remodelling. ${ }^{3}$ In this study, mRNA levels of AREG and HBEGF were decreased in NP tissues and were upregulated by GC treatment. EGR1, another AP-1-related gene, induces c-Jun expression and triggers c-Jun/ AP-1 activation. ${ }^{31}$ Steroid increases expression of EGR1 ${ }^{32}$ EGR1 signals through AP-1/EGF pathways and modulates epithelial remodelling. ${ }^{33}$

This study has several limitations that need to be rectified in future investigations. We recruited a relatively small number of $\mathrm{NP}$ and control subjects in this study. Follow-up studies are needed in a large number of patients to verify this important finding. Another potential shortcoming is the analysis of NP

Table 2 c-Jun protein in GC-naïve and GC-treated nasal polyp epithelium

\begin{tabular}{|c|c|c|c|c|c|c|}
\hline \multirow[b]{2}{*}{ Group } & \multicolumn{3}{|c|}{ c-Jun protein staining* } & \multicolumn{3}{|c|}{ Epithelial integrity $\dagger$} \\
\hline & Weak & Strong & p Value: & Damage & Intact & p Value: \\
\hline GC-naïve NP & 6 & 4 & 0.01 & 8 & 2 & 0.023 \\
\hline GC-treated NP & 0 & 10 & & 2 & 8 & \\
\hline Damaged epithelium† & 6 & 4 & 0.01 & NA & NA & NA \\
\hline Intact epithelium $\dagger$ & 0 & 10 & & NA & NA & \\
\hline
\end{tabular}

*Referring to $c$-Jun protein staining in table 1 (overall score of $\geqslant 6$ defined as "strong" expression and $<6$ as "weak" expression)

$\uparrow$ Referring to epithelium damage in table 1 (grade 0 defined as intact epithelium; grades 1 and 2 defined as damaged epithelium)

$\$ p$ Value obtained by the Fisher exact test.

GC, glucocorticosteroid; NA, not applicable; NP, nasal polyposis. 
versus nasal mucosal control. Since there are various cell types in nasal tissues, some of the findings may reflect the differences in the cellular make-up of the tissues. A further limitation of the current study is that the functional networks and their essential gene-gene interactions are deduced by an array modelling approach which is based on predetermined database knowledge and can only be considered as a source of hypotheses. Functional testing will therefore be needed for the rigorous evaluation of individual molecular interactions inferred by our database approach.

In conclusion, we have explored the molecular mechanisms underlying the pathogenesis of NP and their response to GC treatment. Our data suggest that AP-1 and its related gene network are central molecular effectors of epithelial damage and repair, which can be modulated by GC treatment. Our findings may contribute to a better understanding of the key molecular pathways implicated in the pathogenesis of NP. We hypothesise that these immunoregulatory and remodelling effects elicited by steroids might, at least in part, be mediated by the AP-1 gene network.

Acknowledgements: The authors thank Dr Shanthi Wasser and Dr Li Yang for their assistance in real-time PCR work, and Wen Hong Mei and Dr Yang Yan for technical expertise in histological tissue processing and staining.

Funding: DYW is funded by grants (NMRC/0396/1999 and NMRC/1162/2008) from the National Medical Research Council (NMRC) of Singapore.

Competing interests: None.

Ethics approval: Approval to conduct this study was obtained from the Institutional Review Board of the First Affiliated Hospital, Sun Yet-Sen University and the National University of Singapore.

\section{REFERENCES}

1. Fokkens W, Lund V, Mullol J. European position paper on Rhinosinusitis and Nasal Polyps Group. Rhinology Supp/ 2007;(20):1-136.

2. Jordana M, Nakano K, Nakano A, et al. Nasal polyposis: a model of chronic airways inflammation. In: Busse WW, Holgate ST, eds. Asthma and rhinitis. 2nd ed. Oxford: Blackwell Scientific Publications, 2000:223-31.

3. Watelet JB, Van Zele T, Gjomarkaj M, et al. Tissue remodelling in upper airways: where is the link with lower airway remodelling? Allergy 2006:61:1249-58.

4. Barnes PJ. Corticosteroid effects on cell signalling. Eur Respir J 2006;27:413-26.

5. Mastruzzo C, Greco LR, Nakano K, et al. Impact of intranasal budesonide on immune inflammatory responses and epithelial remodeling in chronic upper airway inflammation. J Allergy Clin Immunol 2003;112:37-44.

6. Wadsworth SJ, Nijmeh HS, Hall IP. Glucocorticoids increase repair potential in a novel in vitro human airway epithelial wounding model. J Clin Immunol 2006;26:376-87.

7. Reddy SP, Mossman BT. Role and regulation of activator protein-1 in toxicant-induced responses of the lung. Am J Physiol Lung Cell Mol Physiol 2002;283:1161-78.

8. Wang J, Homer RJ, Chen 0 , et al. Endogenous and exogenous IL-6 inhibit aeroallergen-induced Th2 inflammation. J Immunol 2000;165:4051-61.

9. Watelet JB, Bachert C, Gevaert $P$, et al. Wound healing of the nasal and paranasal mucosa: a review. Am J Rhinol 2002;16:77-84.
10. Livak KJ, Schmittgen TD. Analysis of relative gene expression data using real-time quantitative PCR and the 2(-Delta Delta C(T)) method. Methods 2001;25:402-8.

11. Wladislavosky-Waserman $\mathbf{P}$, Kern EB, Holley KE, et al. Epithelial damage in nasal polyps. Clin Allergy 1984;14:241-7.

12. Putti TC, To KF, Hsu HC, et al. Expression of epidermal growth factor receptor in head and neck cancers correlates with clinical progression: a multicentre immunohistochemical study in the Asia-Pacific region. Histopathology 2002;41:144-51.

13. Karin M, Liu Z, Zandi E. AP-1 function and regulation. Curr Opin Cell Biol 1997:9:240-6.

14. Grose R. Epithelial migration: open your eyes to c-Jun. Curr Biol 2003;13:678-80.

15. Gallucci RM, Simeonova PP, Matheson JM, et al. Impaired cutaneous wound healing in interleukin-6-deficient and immunosuppressed mice. FASEB $\mathrm{J}$ 2000;14:2525-31.

16. Yamaguchi K, Lantowski A, Dannenberg AJ, et al. Histone deacetylase inhibitors suppress the induction of C-Jun and its target genes including COX-2. J Biol Chem 2005:280:32569-77.

17. Li G, Gustafson-Brown C, Hanks SK, et al. C-Jun is essential for organization of the epidermal leading edge. Dev Cell 2003;4:865-77.

18. Baraniuk JN, Wong G, Ali M, et al. Glucocorticoids decrease c-fos expression in human nasal polyps in vivo. Thorax 1998;53:577-82.

19. Zhou F, Thompson EB. Role of c-jun induction in the glucocorticoid-evoked apoptotic pathway in human leukemic lymphoblasts. Mol Endocrinol 1996;10:306-16.

20. Lee $\mathbf{H}$, Shaw YT, Chiou ST, et al. The effects of glucocorticoid hormone on the expression of c-jun. FEBS Lett 1991;280:134-6.

21. Bolger WE, Joshi AS, Spear S, et al. Gene expression analysis in sinonasal polyposis before and after oral corticosteroids: a preliminary investigation. Otolaryngol Head Neck Surg 2007;137:27-33.

22. Sassone-Corsi $\mathbf{P}$, Sisson JC, Verma IM. Transcriptional autoregulation of the protooncogene fos. Nature 1988;334:314-9.

23. Yang-Yen HF, Chambard JC, Sun YL, et al. Transcriptional interference between CJun and the glucocorticoid receptor: mutual inhibition of DNA binding due to direct protein-protein interaction. Cell 1990;62:1205-15

24. Savla U, Appel HJ, Sporn PH, et al. Prostaglandin E(2) regulates wound closure in airway epithelium. Am J Physiol Lung Cell Mol Physiol 2001;280:421-31.

25. Mullol J, Fernandez-Morata JC, Roca-Ferrer J, et al. Cyclooxygenase 1 and cyclooxygenase 2 expression is abnormally regulated in human nasal polyps. J Allergy Clin Immunol 2002;109:824-30.

26. Dworski RT, Funk CD, Oates JA, et al. Prednisone increases PGH-synthase 2 in atopic humans in vivo. Am J Respir Crit Care Med 1997;155:351-7.

27. Franchimont $\mathbf{N}$, Durant $\mathrm{D}$, Rydziel $\mathrm{S}$, et al. Platelet-derived growth factor induces interleukin-6 transcription in osteoblasts through the activator protein-1 complex and activating transcription factor-2. J Biol Chem 1999;274:6783-9.

28. Solís-Herruzo JA, Rippe RA, Schrum LW, et al. Interleukin-6 increases rat metalloproteinase-13 gene expression through stimulation of activator protein 1 transcription factor in cultured fibroblasts. J Biol Chem 1999:274:30919-26.

29. Homma T, Kato A, Hashimoto N, et al. Corticosteroid and cytokines synergistically enhance toll-like receptor 2 expression in respiratory epithelial cells. Am J Respir Cell Mol Biol 2004;31:463-9.

30. Johnson AC, Murphy BA, Matelis CM, et al. Activator protein-1 mediates induced but not basal epidermal growth factor receptor gene expression. Mol Med 2000;6:17-27.

31. Levkovitz Y, Baraban JM. A dominant negative inhibitor of the Egr family of transcription regulatory factors suppresses cerebellar granule cell apoptosis by blocking c-Jun activation. J Neurosci 2001;21:5893-901.

32. Warburton G, Nares S, Angelov N, et al. Transcriptional events in a clinical model of oral mucosal tissue injury and repair. Wound Repair Regen 2005;13:19-26.

33. Revest JM, Di Blasi F, Kitchener P, et al. The MAPK pathway and Egr-1 mediate stress-related behavioral effects of glucocorticoids. Nat Neurosci 2005;8:664-72.

\section{Keep up to date: sign up for our alerting services}

Find out automatically when an article is published on a specific topic or by a particular author. We can also alert you when an article is cited or if an eLetter or correction is published. You can also choose to be alerted when a new issue is published online [and when we post articles Online First]. Check out the New Content Alerts and Citation tracker from the Online tools section on the home page. 